\title{
ACCURACY ESTIMATE WITH RESPECT TO STATE OF FINITE-DIMENSIONAL APPROXIMATIONS FOR OPTIMIZATION PROBLEMS FOR SEMI-LINEAR ELLIPTIC EQUATIONS WITH DISCONTINUOUS COEFFICIENTS AND SOLUTIONS
}

\author{
A.R. MANAPOVA*，F.V. LUBYSHEV
}

\begin{abstract}
In the work we consider nonlinear optimal control problems for semilinear elliptic equations with discontinuous coefficients and solutions with control in the conjugation boundary conditions. We construct difference approximations for extremum problems and obtain the estimates for approximation accuracy with respect to the state.
\end{abstract}

Keywords: optimal control problem, semi-linear elliptic equations, difference method of solving.

Mathematics Subject Classification: 49J20, 35A35, 35J61, 65N06

\section{INTRODUCTION}

Optimization mathematical models for systems with distributed parameters described by the equations of mathematical physics (EMP) is the most complicated class of problems in the optimization theory, especially for nonlinear control problems. As "nonlinear optimization problems" for EMP we regard those for which the mapping $g \rightarrow u(g)$ from the set of admissible controls $U$ into the space of states $W$ is nonlinear. The nature of specific formulations for problems in optimal control of distributed systems depends essentially on many factors: how the control is involved (into the free terms of state equations or into the coefficients of equations); whether the states of a system are described by linear or nonlinear EMP; what is the structure of set of admissible controls and target functionals; what is the smoothness of state ensured by a given apriori smoothness of initial data and of control and so forth. Nowadays linear control systems with sufficiently smooth initial data and state functions of controls are studied in most details. A special interest both for practical and theoretical points of view is related with a physical and mathematical formulation of optimal control problems, in which, due to a nature of a studied physical process, the states are described by nonlinear EMP with discontinuous coefficients, and moreover, originally in their physical and mathematical formulation, solutions to EMP admit discontinuities.

The issue of numerical solving of optimal control problems leads one to the need of approximating them by simpler problems, namely, by "finite-dimensional problems". A correctly

A.R. Manapova, F.V. Lubyshev, Accuracy estimate With Respect to state of FiniteDIMENSIONAL APPROXIMATIONS FOR OPTIMIZATION PROBLEMS FOR SEMI-LINEAR ELLIPTIC EQUATIONS WITH DisCONTINUOUS COEFFICIENTS AND SOLUTIONS.

(C) Manapova A.R., Lubyshev F.V. 2014.

* The work was supported by a grant of the Republic of Bashkortostan awarded within the contest 2014 of scientific works of young scientists and youth scientific teams.

Submitted January 14, 2014. 
constructed approximation allows one to obtain instructive results of qualitative and quantitative character on the studied process. Basic issues in the approximation problem are "constructing" approximations, convergence of approximations w.r.t. state, functional, control, regularization of approximations [1]-[5]. For systems with distributed parameters, constructing and studying approximations were mainly performed for linear optimal control problems, and with sufficiently smooth coefficients in EMP and states. Here actual issues are "constructing" finite-dimensional approximations and studying their convergence for optimal control problems described by nonlinear EMP with discontinuous coefficients and solutions (states). We observe that difference schemes for equations with discontinuous coefficients but continuous flow and solution (with with perfect-contact matching condition) were constructed and studied in [6], [7] for EMP with classical solutions of certain smoothness. The convergence of difference schemes for parabolic equations with discontinuous coefficients and solutions in classical formulation of the problems with sufficiently smooth solutions were studied in works [8], [9]. We also mention that optimization issues were not considered in these works.

In the present work, in the field related to [1]-[5], [10]-[13], we consider mathematical models of nonlinear optimal control problems described by semilinear elliptic equations in inhomogeneous anisotropic media with discontinuous coefficients and solutions (states) and imperfectcontact matching condition [6], [14]. A coefficient in the conjugation boundary condition serves as a control. We construct difference scheme of extremum problems and establish estimates for approximations convergence rate w.r.t. state.

In terms of termal physics the formulated problem can be interpreted as a problem on optimal control by the coefficient in the boundary condition of heat-conducting media conjugation. At that, this coefficient characterizes the thermal resistance of nonideal contact of heterogeneous media [6], [14].

\section{Formulation of PROBlems}

Let $\Omega=\left\{r=\left(r_{1}, r_{2}\right) \in \mathbb{R}^{2}: 0 \leqslant r_{\alpha} \leqslant l_{\alpha}, \alpha=1,2\right\}$ be a rectangle in $\mathbb{R}^{2}$ with boundary $\partial \Omega=\Gamma$. Suppose that domain $\Omega$ is paritioned by the line $r_{1}=\xi$, where $0<\xi<l_{1}$ ("internal contact boundary" $\bar{S}=\left\{r_{1}=\xi, 0 \leqslant r_{2} \leqslant l_{2}\right\}$, where $0<\xi<l_{1}$ ) into the subdomains $\Omega_{1} \equiv \Omega^{-}=\left\{0<r_{1}<\xi, \quad 0<r_{2}<l_{2}\right\}$ and $\Omega_{2} \equiv \Omega^{+}=\left\{\xi<r_{1}<l_{1}, \quad 0<r_{2}<l_{2}\right\}$ (into left and right subdomains $\Omega_{1}$ and $\Omega_{2}$, respectively) with boundaries $\partial \Omega_{1} \equiv \partial \Omega^{-}$and $\partial \Omega_{2} \equiv \partial \Omega^{+}$. Domain $\Omega$ is the union of domains $\Omega_{1}$ and $\Omega_{2}$ and internal points of "contact" boundary $\bar{S}$ of subdomains $\Omega_{1}$ and $\Omega_{2}$, while $\partial \Omega$ is the external boundary of domain $\Omega$. By $\bar{\Gamma}_{k}$ we denote the boundaries of domains $\Omega_{k}$ without $S, k=1,2$. So, $\partial \Omega_{k}=\bar{\Gamma}_{k} \cup S$, where parts $\Gamma_{k}, k=1,2$, are open non-empty subsets in $\partial \Omega_{k}, k=1,2 ; \bar{\Gamma}_{1} \cup \bar{\Gamma}_{2}=\partial \Omega=\Gamma$. By $n_{\alpha}, \alpha=1,2$ we indicate outward normal to boundary $\partial \Omega_{\alpha}$ of domain $\Omega_{\alpha}, \alpha=1,2$. Let $n=n(x)$ be the unit normal to $S$ at a point $x \in S$ oriented, for instance, so that normal $n$ is outward for $S$ w.r.t. domain $\Omega_{1}$, i.e., normal $n$ is directed inside domain $\Omega_{2}$. In what follows, while formulating boundary value problems for the states of control processes, $S$ stands for the line along which the coefficients and solutions of boundary value problems are discontinuous, while inside domains $\Omega_{1}$ and $\Omega_{2}$ they possess certain smoothness.

Suppose that the conditions of a controlled physical process allow us to model it in the domain $\Omega=\Omega_{1} \cup \Omega_{2} \cup S$ formed by two parts (subdomains) $\Omega_{1}$ and $\Omega_{2}$ and partitioned by internal boundary $S$ and this process is described by the following Dirichlet problem for the semilinear elliptic equations with discontinuous coefficients and solutions:

To find a function $u(x)$ defined on $\bar{\Omega}$ as $u(x)=u_{1}(x), x \in \bar{\Omega}_{1} \equiv \Omega^{-}, u(x)=u_{2}(x)$, $x \in \bar{\Omega}_{2}=\Omega^{+}$, where components $u_{k}, k=1,2$, satisfy the conditions: 
1) functions $u_{k}(x), k=1,2$, defined on $\bar{\Omega}_{k}=\Omega_{k} \cup \partial \Omega_{k}, k=1,2$, satisfy the equations

$$
L_{k} u_{k}=-\sum_{\alpha=1}^{2} \frac{\partial}{\partial x_{\alpha}}\left(k_{\alpha}^{(k)}(x) \frac{\partial u_{k}}{\partial x_{\alpha}}\right)+d_{k}(x) q_{k}\left(u_{k}\right)=f_{k}(x), \quad \text { in } \quad \Omega_{k}, k=1,2,
$$

and the conditions

where $\bar{\Gamma}_{k}=\partial \Omega_{k} \backslash S$.

$$
u_{k}(x)=0, \quad x \in \bar{\Gamma}_{k}, k=1,2,
$$

2) Unknown functions $u_{k}(x), k=1,2$, obey also additional conditions on $S$ which is the boundary of discontinuity of the coefficients and solution and these conditions allow one to "glue" solutions $u_{1}(x)$ and $u_{2}(x)$ along contact boundary $S$ of domains $\Omega_{1}$ and $\Omega_{2}$. These conditions read as follows

$$
G(x)=k_{1}^{(1)}(x) \frac{\partial u_{1}}{\partial x_{1}}=k_{1}^{(2)}(x) \frac{\partial u_{2}}{\partial x_{1}}=\theta\left(x_{2}\right)\left(u_{2}(x)-u_{1}(x)\right), \quad x \in S .
$$

If we introduce the functions

$$
\begin{aligned}
& u(x)= \begin{cases}u_{1}(x), & x \in \Omega_{1} ; \\
u_{2}(x), & x \in \Omega_{2},\end{cases} \\
& q(\xi)= \begin{cases}q_{1}\left(\xi_{1}\right), & \xi_{1} \in \mathbb{R} ; \\
q_{2}\left(\xi_{2}\right), & \xi_{2} \in \mathbb{R},\end{cases} \\
& k_{\alpha}(x), d(x), f(x)=\left\{\begin{array}{ll}
k_{\alpha}^{(1)}(x), d_{1}(x), f_{1}(x), & x \in \Omega_{1} ; \\
k_{\alpha}^{(2)}(x), d_{2}(x), f_{2}(x), & x \in \Omega_{2},
\end{array} \quad \alpha=1,2,\right.
\end{aligned}
$$

problem $(1)=1 \mathrm{a})+(1 \mathrm{~b})+1 \mathrm{c})$ can be rewritten in a more compact form:

To find a function $u(x)$ defined on $\bar{\Omega}$ and satisfying the equation

$$
L u(x)=-\sum_{\alpha=1}^{2} \frac{\partial}{\partial x_{\alpha}}\left(k_{\alpha}(x) \frac{\partial u}{\partial x_{\alpha}}\right)+d(x) q(u)=f(x), \quad x \in \Omega_{1} \cup \Omega_{2},
$$

and conditions $u(x)=0, \quad x \in \partial \Omega=\bar{\Gamma}_{1} \cup \bar{\Gamma}_{2}$,

$$
\left[k_{1}(x) \frac{\partial u}{\partial x_{1}}\right]=0, \quad G(x)=\left(k_{1}(x) \frac{\partial u}{\partial x_{1}}\right)=\theta\left(x_{2}\right)[u], \quad x \in S .
$$

Here $[u]=u_{2}(x)-u_{1}(x)$ is the jump of function $u(x)$ on $S ; k_{\alpha}(x), \alpha=1,2, d(x), f(x)$ are known functions defined in different ways in $\Omega_{1}$ and $\Omega_{2}$ and having a jump discontinuity on $S ; q_{\alpha}\left(\xi_{\alpha}\right), \alpha=1,2$, are given functions defined as $\xi_{\alpha} \in \mathbb{R}, \alpha=1,2 ; \theta(x) \equiv g(x), x \in S$, is a control. For the described functions, we assume:

$k_{\alpha}(x) \in W_{\infty}^{1}\left(\Omega_{1}\right) \times W_{\infty}^{1}\left(\Omega_{2}\right), \quad \alpha=1,2, \quad d(x) \in L_{\infty}\left(\Omega_{1}\right) \times L_{\infty}\left(\Omega_{2}\right), \quad f(x) \in L_{2}\left(\Omega_{1}\right) \times L_{2}\left(\Omega_{2}\right) ;$ $0<\nu \leqslant k_{\alpha}(x) \leqslant \bar{\nu}, \alpha=1,2,0 \leqslant d_{0} \leqslant d(x) \leqslant \bar{d}_{0}, x \in \Omega_{1} \cup \Omega_{2} ; \nu, \bar{\nu}, d_{0}, \bar{d}_{0}$ are given constants; functions $q_{\alpha}\left(\xi_{\alpha}\right)$ are defined on $\mathbb{R}$ with values in $\mathbb{R}$ and satisfy the conditions: $q_{\alpha}(0)=0$, $0<q_{0} \leqslant\left(q_{\alpha}\left(\xi_{\alpha}\right)-q_{\alpha}\left(\bar{\xi}_{\alpha}\right)\right) /\left(\xi_{\alpha}-\bar{\xi}_{\alpha}\right) \leqslant L<\infty$ for each $\xi_{\alpha}, \bar{\xi}_{\alpha} \in \mathbb{R}, \xi_{\alpha} \neq \bar{\xi}_{\alpha}, \alpha=1,2$.

We introduce the set of admissible controls

$$
U=\left\{g(x)=\theta(x) \in L_{2}(S)=H: 0<g_{0} \leqslant g(x) \leqslant \bar{g}_{0} \text { a.e. on } S\right\},
$$

where $L_{2}(S)=H$ is the space of controls, $U \subset H, g_{0}, \bar{g}_{0}$ are given numbers.

We define a target functional $J: U \rightarrow \mathbb{R}^{1}$ as

$$
g \rightarrow J(g)=\int_{\Omega_{1}}\left|u\left(r_{1}, r_{2} ; g\right)-u_{0}^{(1)}(r)\right|^{2} d \Omega_{1}=I(u(r ; g)),
$$


where $u_{0}^{(1)} \in W_{2}^{1}\left(\Omega_{1}\right)$ is a given function.

The optimal control problem is to find a control $g_{*} \in U$ minimizing functional $g \rightarrow J(g)$ on set $U \subset H$, namely, on solutions $u(r)=u(r ; g)$ to problem (1) obeying all admissible controls $g=\theta \in U$, we need to minimize functional $(3)$.

We introduce the space $V\left(\Omega^{(1,2)}\right), \Omega^{(1,2)}=\Omega_{1} \cup \Omega_{2}$, of pairs of functions $u(x)=\left(u_{1}(x), u_{2}(x)\right)$ : $V\left(\Omega^{(1,2)}\right)=\left\{u(x)=\left(u_{1}(x), u_{2}(x)\right) \in W_{2}^{1}\left(\Omega_{1}\right) \times W_{2}^{1}\left(\Omega_{2}\right)\right\}$, where $W_{2}^{1}\left(\Omega_{k}\right), k=1,2$, are Sobolev spaces of functions defined in subdomains $\Omega_{k}$ with boundaries $\partial \Omega_{k}, k=1,2$, respectively, and with the norms [15]-[19]:

$$
\left\|u_{k}\right\|_{W_{2}^{1}\left(\Omega_{k}\right)}^{2}=\int_{\Omega_{k}}\left[\sum_{\alpha=1}^{2}\left(\frac{\partial u_{k}}{\partial x_{\alpha}}\right)^{2}+u_{k}^{2}\right] d \Omega_{k}, \quad k=1,2 .
$$

Space $V=V\left(\Omega^{(1,2)}\right)$ equipped with the scalar product $(u, \vartheta)_{V}=\sum_{k=1}^{2}\left(u_{k}, \vartheta_{k}\right)_{W_{2}^{1}\left(\Omega_{k}\right)}$ and the norm $\|u\|_{V}^{2}=\sum_{k=1}^{2}\left\|u_{k}\right\|_{W_{2}^{1}\left(\Omega_{k}\right)}^{2}$ is a Hilbert one.

One can show that in Hilbert space $V\left(\Omega^{(1,2)}\right)$ one can introduce the equivalent norm

$$
\|u\|_{*}^{2}=\sum_{k=1}^{2} \int_{\Omega_{k}} \sum_{\alpha=1}^{2}\left(\frac{\partial u_{k}}{\partial x_{\alpha}}\right)^{2} d \Omega_{k}+\sum_{k=1}^{2} \int_{\Gamma_{k}} u_{k}^{2} d \Gamma_{k}+\int_{S}[u]^{2} d S
$$

where $[u]=u_{2}(x)-u_{1}(x)=u^{+}(x)-u^{-}(x)$ is the jump of function $u(x)$ on $S$. Here $u_{2}(x)=$ $u^{+}(x), x \in S$, and $u_{1}(x)=u^{-}(x), x \in S$, are the traces of function $u(x)$ on $S$ while approaching from $\Omega_{2}=\Omega^{+}$and from $\Omega_{1}=\Omega^{-}$, respectively. It it clear that the condition $u(x) \in V\left(\Omega^{(1,2)}\right)$ implies the boundedness for the embedding of spaces $W_{2}^{1}\left(\Omega_{k}\right), k=1,2$, into spaces $L_{2}\left(\partial \Omega_{k}\right)$, $k=1,2$, since $\Omega_{1}$ and $\Omega_{2}$ are domains with Lipschitz boundaries $\partial \Omega_{1}$ and $\partial \Omega_{2}$. In particular, the condition $u(x) \in V\left(\Omega^{(1,2)}\right)$ yields $[u(x)] \in L_{2}(S)$, since here the theorem on traces [15]-[19] holds true for each of sides $S^{+}, S^{-}$of the contact boundary $S$ (the restriction operator from $W_{2}^{1}\left(\Omega^{ \pm}\right)$into $L_{2}(S)$ is continuous). We also observe that applying the theorem on traces to $\Omega_{1}$ and $\Omega_{2}$ allows us to define two traces for each function $u(x) \in V\left(\Omega^{(1,2)}\right)$ by means of restriction operators on $S^{ \pm}$. On the other hand, if $u \in V\left(\Omega^{(1,2)}\right)$, its traces on different sides of $S$ (while approaching from $\Omega_{1}$ and from $\Omega_{2}$ ) are different in the general situation. The restrictions of function $u(x)$ on domains $\Omega_{k}, k=1,2:\left.u\right|_{\Omega_{k}}, k=1,2$, belong respectively to spaces $W_{2}^{1}\left(\Omega_{k}\right)$, $k=1,2$, but function $u$ is not an element of space $W_{2}^{1}(\Omega)$ since it has a jump on set $S$ while passing from $\Omega_{1}$ into $\Omega_{2}: \delta(x)=u_{2}(x)-u_{1}(x)=u^{+}(x)-u^{-}(x), x \in S$. We note that the criterion of belonging $\vartheta(x) \in W_{2}^{1}(\Omega)=W_{2}^{1}\left(\Omega_{1} \cup \Omega_{2} \cup S\right)$ is the gluing condition $\vartheta_{k}(x) \in W_{2}^{1}\left(\Omega_{k}\right)$, $k=1,2 ;\left.\vartheta_{1}(x)\right|_{S}=\left.\vartheta_{2}(x)\right|_{S}$ (see, for instance, [19]).

Since $\Omega_{k}$ are domains with Lipshitz boundaries $\partial \Omega_{k}, k=1,2$, and $\Gamma_{1}$ and $\Gamma_{2}$ are respectively their open parts (pieces of boundaries $\partial \Omega_{1}$ and $\partial \Omega_{2}$ ) of positive measures mes $\Gamma_{k}>0, k=1,2$, then [20] there exist some constants $C_{1}$ and $C_{2}$ depending only on domains $\Omega_{k}, k=1,2$, and pieces $\Gamma_{1}$ and $\Gamma_{2}$, respectively, so that for each function $u_{k}(x) \in W_{2}^{1}\left(\Omega_{k}\right), k=1,2$, the relations

$$
\left\|u_{k}\right\|_{W_{2}^{1}\left(\Omega_{k}\right)}^{2} \leqslant C_{k}^{2}\left[\int_{\Omega_{k}} \sum_{\alpha=1}^{2}\left(\frac{\partial u_{k}}{\partial x_{\alpha}}\right)^{2} d \Omega_{k}+\int_{\Gamma_{k}} u_{k}^{2} d \Gamma_{k}\right], \quad k=1,2,
$$

hold true. Since for considered domains $\Omega_{k}, k=1,2$, the embeddings of spaces $W_{2}^{1}\left(\Omega_{k}\right), k=$ 1,2 , into spaces $L_{2}\left(\partial \Omega_{k}\right), k=1,2$, are bounded, there exist constants $C_{3}$ and $C_{4}$ independent of function $u_{k}(x)$ such that for each function $u_{k}(x) \in W_{2}^{1}\left(\Omega_{k}\right)$ the estimates

$$
\left\|u_{k}\right\|_{L_{2}\left(\partial \Omega_{k}\right)}^{2} \leqslant C_{k+2}^{2}\left\|u_{k}\right\|_{W_{2}^{1}\left(\Omega_{k}\right)}^{2}, \quad k=1,2
$$


hold true [16], [17] and these estimates are implied by the theorems on embedding of $W_{2}^{1}\left(\Omega_{k}\right)$ into $L_{2}\left(\partial \Omega_{k}\right)$.

Let $\stackrel{\circ}{\Gamma}_{k}$ be a part of $\partial \Omega_{k}$. By $W_{2}^{1}\left(\Omega_{k} ; \stackrel{\circ}{\Gamma}_{k}\right)$ we denote the closed subspace of space $W_{2}^{1}\left(\Omega_{k}\right)$, whose dense set is that of functions in $C^{1}\left(\bar{\Omega}_{k}\right)$ vanishing in the vicinity of $\stackrel{\circ}{\Gamma}_{k} \subset \partial \Omega_{k}, k=$ 1,2. As $\stackrel{\circ}{\Gamma}_{k}$, we choose some pieces of boundary $\partial \Omega_{k}$; of course, we do not consider the case when one of pieces $\stackrel{\circ}{\Gamma}_{k}$ degenerates to a point; $W_{2}^{1}\left(\Omega_{k} ; \stackrel{\circ}{\Gamma} k\right)$ coincides with $W_{2}^{1}\left(\Omega_{k}\right)$ as $\stackrel{\circ}{\Gamma}_{k}=\emptyset$; $W_{2}^{1}\left(\Omega_{k} ; \stackrel{\circ}{\Gamma}_{k}\right)=\stackrel{0}{W} \underset{2}{1}\left(\Omega_{k}\right)$ as $\stackrel{\circ}{\Gamma}_{k}=\partial \Omega_{k}$. We note that for elements $u_{k}(x) \in W_{2}^{1}\left(\Omega_{k} ; \stackrel{\circ}{\Gamma}_{k}\right)$ the inequality

$$
\int_{\Omega_{k}} u_{k}^{2}(x) d \Omega_{k} \leqslant C_{k+4}\left(\Omega_{k}, \stackrel{\circ}{\Gamma} k\right) \int_{\Omega_{k}} \sum_{\alpha=1}^{2}\left(\frac{\partial u_{k}}{\partial x_{\alpha}}\right)^{2} d \Omega_{k}, \quad k=1,2
$$

holds true [16] with a constant $C_{k+4}\left(\Omega_{k}, \stackrel{\circ}{\Gamma}_{k}\right)$ depending only on $\Omega_{k}$ and $\stackrel{\circ}{\Gamma}_{k}$. At that, the area of piece $\stackrel{\circ}{\Gamma}_{k}$ of surface $\partial \Omega_{k}$ must be positive: mes $\stackrel{\circ}{\Gamma}_{k}>0$.

We introduce the space $\stackrel{\circ}{V}_{\Gamma_{1}, \Gamma_{2}}\left(\Omega^{(1,2)}\right)$ of pairs of functions $u(x)=\left(u_{1}(x), u_{2}(x)\right): \stackrel{\circ}{V}_{\Gamma_{1}, \Gamma_{2}}$ $\left(\Omega^{(1,2)}\right)=\left\{u(x)=\left(u_{1}(x), u_{2}(x)\right) \in W_{2}^{1}\left(\Omega_{1} ; \Gamma_{1}\right) \times W_{2}^{1}\left(\Omega_{2} ; \Gamma_{2}\right)\right\}$ with the norm

$$
\|u\|_{\substack{\circ \\ V_{\Gamma_{1}}, \Gamma_{2}}}^{2}=\sum_{k=1}^{2} \int_{\Omega_{k}} \sum_{\alpha=1}^{2}\left(\frac{\partial u_{k}}{\partial x_{\alpha}}\right)^{2} d \Omega_{k}+\int_{S}[u]^{2} d S .
$$

By a solution to direct problem (1) for a fixed control $g(x)=\theta(x) \in U$ we mean a function $u(x) \equiv u(x ; g) \in \stackrel{\circ}{V}_{\Gamma_{1}, \Gamma_{2}}\left(\Omega^{(1,2)}\right)$ satisfying the identity

$$
\begin{aligned}
Q(u, \vartheta) & =\int_{\Omega_{1} \cup \Omega_{2}}\left[\sum_{\alpha=1}^{2} k_{\alpha}(x) \frac{\partial u}{\partial x_{\alpha}} \frac{\partial \vartheta}{\partial x_{\alpha}}+d(x) q(u) \vartheta\right] d \Omega_{0}+\int_{S} \theta(x)[u][\vartheta] d S \\
& =\int_{\Omega_{1} \cup \Omega_{2}} f(x) \vartheta d \Omega_{0}=l(\vartheta) .
\end{aligned}
$$

for each $\vartheta \in \stackrel{\circ}{V}_{\Gamma_{1}, \Gamma_{2}}\left(\Omega^{(1,2)}\right)$.

The following theorem holds true.

Theorem 1. For each $g \in U$ there exists the unique generalized solution $u(x)=$ $u(x ; g) \in \stackrel{\circ}{V}_{\Gamma_{1}, \Gamma_{2}}\left(\Omega^{(1,2)}\right)$ to problem (1) determined by integral identity (5). At that,

$$
\|u(x, g)\|_{{\stackrel{\circ}{\Gamma_{1}, \Gamma_{2}}}^{\circ}} \leqslant C_{11} \sum_{k=1}^{2}\left\|f_{k}(x)\right\|_{L_{2}\left(\Omega_{k}\right)}=C_{12}, \quad \forall g \in U
$$

where $C_{11}=$ Const $>0$.

Proof. The proof of the theorem is based on the theory of monotone operators [17], [18], [21]. At that, one should employ substantially above introduced Hilbert spaces $V\left(\Omega^{(1,2)}\right), \stackrel{\circ}{V}_{\Gamma_{1} \Gamma_{2}}\left(\Omega^{(1,2)}\right)$ and the equivalent norms as well as the inequalities. 
We begin with identity (5). It is easy to make sure that the chain of inequalities

$$
\begin{aligned}
|Q(u, \vartheta)| \leqslant & \int_{\Omega_{1} \cup \Omega_{2}}\left[\bar{\nu} \sum_{\alpha=1}^{2}\left|\frac{\partial u}{\partial x_{\alpha}}\right|\left|\frac{\partial \vartheta}{\partial x_{\alpha}}\right|+\bar{d}_{0} L_{q}|u||\vartheta|\right] d \Omega_{0}+\bar{\theta}_{0} \iint_{S}|[u]||[\vartheta]| d S \\
\leqslant & \max \left\{\bar{\nu}, \bar{d}_{0} L_{q}, \bar{g}_{0}\right\}\left[\sum_{\alpha=1}^{2} \int_{\Omega_{1} \cup \Omega_{2}}\left|\frac{\partial u}{\partial x_{\alpha}}\right|^{2} d \Omega_{0}+\int_{\Omega_{1} \cup \Omega_{2}} u^{2} d \Omega_{0}+\int_{S}[u]^{2} d S\right]^{1 / 2} \\
& \cdot\left[\sum_{\alpha=1}^{2} \int_{\Omega_{1} \cup \Omega_{2}}\left|\frac{\partial \vartheta}{\partial x_{\alpha}}\right|^{2} d \Omega_{0}+\int_{\Omega_{1} \cup \Omega_{2}} \vartheta^{2} d \Omega_{0}+\int_{S}[\vartheta]^{2} d S\right]^{1 / 2}
\end{aligned}
$$

hold true. Employing inequality (4), one can easily establish the estimate

$$
\begin{aligned}
& \sum_{\alpha=1}^{2} \int_{\Omega_{1} \cup \Omega_{2}}\left|\frac{\partial u}{\partial x_{\alpha}}\right|^{2} d \Omega_{0}+\int_{\Omega_{1} \cup \Omega_{2}} u^{2} d \Omega_{0}+\int_{S}[u]^{2} d S=\sum_{k=1}^{2}\left\|u_{k}\right\|_{W_{2}^{1}\left(\Omega_{k}\right)}^{2}+\int_{S}[u]^{2} d S \\
& \leqslant C_{7}^{2}\left[\sum_{k=1}^{2} \int_{\Omega_{k}} \sum_{\alpha=1}^{2}\left|\frac{\partial u_{k}}{\partial x_{\alpha}}\right|^{2} d \Omega_{k}+\sum_{k=1}^{2} \int_{\Gamma_{k}} u_{k}^{2} d \Gamma_{k}+\int_{S}[u]^{2} d S\right]=C_{7}^{2}\|u\|_{*}^{2},
\end{aligned}
$$

where $C_{7}^{2}=\max \left\{1, \max \left(C_{1}^{2}, C_{2}^{2}\right\}\right.$. Taking into consideration (8) and since $u_{k}(x)=0$ on $\Gamma_{k}$, $k=1,2$, by (7) we obtain the estimate

$$
|Q(u, \vartheta)| \leqslant \max \left\{\bar{\nu}, \bar{d}_{0} L_{q}, \bar{g}_{0}\right\} C_{7}^{2}\|u\|_{{\stackrel{\circ}{\Gamma_{1}, \Gamma_{2}}}^{\circ}}\|\vartheta\|_{{\stackrel{\circ}{\Gamma_{1}, \Gamma_{2}}}^{\circ}}, \quad \forall u, \vartheta \in \stackrel{\circ}{V_{\Gamma_{1}, \Gamma_{2}}} .
$$

Hence, for each fixed $u \in \stackrel{\circ}{V}_{\Gamma_{1}, \Gamma_{2}}$ form $Q(u, \vartheta)$ defines a linear functional in Hilbert space $\stackrel{\circ}{V}_{\Gamma_{1}, \Gamma_{2}}$ boundary w.r.t. $\vartheta \in \stackrel{\circ}{V}_{\Gamma_{1}, \Gamma_{2}}$ depending on function $u \in \stackrel{\circ}{V}_{\Gamma_{1}, \Gamma_{2}}$. We indicate this functional as $\Phi=A u \in \stackrel{\circ}{V}_{\Gamma_{1}, \Gamma_{2}}$. It is defined by the relation

$$
\langle\Phi, \vartheta\rangle=\langle A u, \vartheta\rangle=(A u, \vartheta)_{{\stackrel{\circ}{\Gamma_{1}, \Gamma_{2}}}}=Q(u, \vartheta), \quad \forall \vartheta \in \stackrel{\circ}{V}_{\Gamma_{1}, \Gamma_{2}}
$$

where operator $A: \stackrel{\circ}{V}_{\Gamma_{1}, \Gamma_{2}} \rightarrow \stackrel{\circ}{V}_{\Gamma_{1}, \Gamma_{2}}$ maps each element $u \in \stackrel{\circ}{V}_{\Gamma_{1}, \Gamma_{2}}$ into a linear continuous functional $\Phi=A u$ in space $\stackrel{\circ}{V}_{\Gamma_{1}, \Gamma_{2}}$ so that the value of functional $\Phi=A u$ on element $\vartheta \in \stackrel{\circ}{V}_{\Gamma_{1}, \Gamma_{2}}$ is defined by relation (9).

Let us consider the right hand side of identity $(5)$. We let $\langle F, \vartheta\rangle=l(\vartheta)$. It is easy to prove that the estimate

$$
|\langle F, \vartheta\rangle|=|l(\vartheta)| \leqslant \sum_{k=1}^{2}\left\|f_{k}\right\|_{L_{2}\left(\Omega_{k}\right)} \cdot\left\|\vartheta_{k}\right\|_{L_{2}\left(\Omega_{k}\right)} \leqslant C_{8} \sum_{k=1}^{2}\left\|f_{k}\right\|_{L_{2}\left(\Omega_{k}\right)} \cdot\left\|\vartheta_{k}\right\|_{{\stackrel{\circ}{\Gamma_{1}, \Gamma_{2}}}^{\circ}}
$$

is valid, where $C_{8}=\sqrt{2} \max \left\{C_{1}, C_{2}\right\}$. Thus, functional $F$ defined by means of the formula $\langle F, \vartheta\rangle=l(\vartheta)$ is bounded on $\stackrel{\circ}{V}_{\Gamma_{1}, \Gamma_{2}}$ and moreover, this functional is linear and therefore, $F \in \stackrel{\circ}{V}_{\Gamma_{1}, \Gamma_{2}}$. Thus, identity (5) casts into the form $\langle A u, \vartheta\rangle=\langle F, \vartheta\rangle, \forall \vartheta \in \stackrel{\circ}{V}_{\Gamma_{1}, \Gamma_{2}}$, which by the arbitrariness of $\vartheta \in \stackrel{\circ}{V}_{\Gamma_{1}, \Gamma_{2}}$ yields the equation $A u=F$.

Let us show that there exists the unique solution $u \in \stackrel{\circ}{V}_{\Gamma_{1}, \Gamma_{2}}$ satisfying identity (5). Thanks to Browder theorem [21], it is sufficient to prove the continuity and strong monotonicity for operator $A$. It is easy to make sure that the estimate $\langle A u-A \vartheta, u-\vartheta\rangle \geqslant \min \left\{\nu, g_{0}\right\}\|u-\vartheta\|_{V_{\Gamma_{1}, \Gamma_{2}}}^{2}$, $\forall u, \vartheta \in \stackrel{\circ}{V}_{\Gamma_{1}, \Gamma_{2}}$, holds true. It means that operator $A: \stackrel{\circ}{V}_{\Gamma_{1}, \Gamma_{2}} \rightarrow \stackrel{\circ}{V}_{\Gamma_{1}, \Gamma_{2}}$ is strongly monotone. Let us prove the continuity, namely, Lipschitz continuity for operator $A$. It is easy 
to make sure that the estimate $|\langle A u-A \vartheta, \eta\rangle| \leqslant C_{9}\|u-\vartheta\|_{{\stackrel{\circ}{\Gamma_{1}, \Gamma_{2}}}^{\circ}}\|\eta\|_{V_{\Gamma_{1}, \Gamma_{2}}}, \forall u, \vartheta, \eta \in{\stackrel{\circ}{\Gamma_{1}, \Gamma_{2}}}$, $C_{9}=\max \left\{\bar{\nu}, \bar{d}_{0} L_{q}, \bar{g}_{0}\right\} C_{7}^{2}$, is valid. Hence,

$$
\|A u-A \vartheta\|_{V_{\Gamma_{1}, \Gamma_{2}}^{\circ}}=\sup _{\eta \neq 0} \frac{|\langle A u-A \vartheta, \eta\rangle|}{\|\eta\|_{{\stackrel{\circ}{\Gamma_{1}, \Gamma_{2}}}^{\circ}}} \leqslant C_{9}\|u-\vartheta\|_{V_{\Gamma_{1}, \Gamma_{2}}^{\circ}}, \quad \forall u, \vartheta \in{\stackrel{\circ}{\Gamma_{\Gamma_{1}}, \Gamma_{2}}},
$$

i.e., operator $A$ is Lipschitz continuous. Hence, the hypothesis of Browder theorem is satisfied, and thus, the equation $A u=F$ is uniquely solvable.

Employing the coercitivity $\left(\stackrel{\circ}{V}_{\Gamma_{1}, \Gamma_{2}}\right.$ ellipticity) of form $Q(u, \vartheta)$ on $\stackrel{\circ}{V}_{\Gamma_{1}, \Gamma_{2}}: Q(u, u) \geqslant$ $C_{10}\|u\|_{{\stackrel{\circ}{\Gamma_{1}, \Gamma_{2}}}^{2}}^{2}, \forall u \in \stackrel{\circ}{V}_{\Gamma_{1}, \Gamma_{2}}, C_{10}=\min \left\{\nu, g_{0}\right\}$, and estimate $(10)$, we obtain $C_{10}\|u\|_{V_{\Gamma_{1}, \Gamma_{2}}}^{2} \leqslant$ $Q(u, u)=l(u) \leqslant C_{8} \sum_{k=1}^{2}\left\|f_{k}\right\|_{L_{2}\left(\Omega_{k}\right)} \cdot\|u\|_{{\stackrel{\circ}{\Gamma_{1}, \Gamma_{2}}}}$. It yields estimate (6) with constant $C_{11}=$ $C_{8} \cdot C_{10}^{-1}$.

In what follows, while studying the convergence of difference approximations w.r.t. state for optimal control problems, we make the following assumption for the smoothness similar to that made in work [22] in studying difference schemes for a problem with the same conjugation conditions. We assume that the solution to boundary value problem (1) belongs to $W_{2}^{2}\left(\Omega_{1}\right) \times$ $W_{2}^{2}\left(\Omega_{2}\right)$, namely, to space $\stackrel{\circ}{V}_{\Gamma_{1}, \Gamma_{2}}\left(\Omega^{(1,2)}\right)=\stackrel{\circ}{V}_{\Gamma_{1}, \Gamma_{2}}\left(\Omega^{(1,2)}\right) \cap\left\{u=\left(u_{1}, u_{2}\right) \in W_{2}^{2}\left(\Omega_{1}\right) \times W_{2}^{2}\left(\Omega_{2}\right)\right\}$, and for each fixed control $g \in U$ the estimate

$$
\sum_{k=1}^{2}\left\|u_{k}(x, g)\right\|_{W_{2}^{2}\left(\Omega_{k}\right)} \leqslant M \sum_{k=1}^{2}\left\|f_{k}(x)\right\|_{L_{2}\left(\Omega_{k}\right)}, \quad \forall g \in U, \quad \text { where } \quad M=\text { Const }>0,
$$

is valid.

Remark 1. Hereinafter by $C, C_{k}, k=\overline{1,7}, M$ we denote various positive constants independent of solution $u(r) \equiv u(r ; g)$ and control $g \in U$ (of grid solution $y(x) \equiv y\left(x ; \Phi_{h}\right)$ and grid control $\left.\Phi_{h} \in U_{h}\right)$.

\section{Difference approximation of optimization problems. Apriori estimates FOR ERRORS AND CONVERGENCE RATE OF GRID EXTREMUM PROBLEM W.R.T. STATE}

Because of numerical solving of optimal control problems an essential interest is arisen by the issue on approximations of infinite-dimensional optimization problems (1)-(3) by a sequence of finite-dimensional optimal control problems. In what follows we construct approximations for problems on the base of the grid method (see [5], 6]) and study the convergence of these approximation w.r.t. state as the step $h$ of the discretization mesh tends to zero. To approximate problems (1)-(3), we shall employ some grids on $\left[0, l_{\alpha}\right], \alpha=1,2$, and in $\bar{\Omega}$. We introduce one-dimensional non-uniform grids w.r.t. $x_{1}$ and $x_{2}: \widehat{\omega}_{\alpha}=\left\{x_{\alpha}^{\left(i_{\alpha}\right)} \in\left[0, l_{\alpha}\right]: i_{\alpha}=\overline{0, N_{\alpha}}, x_{\alpha}^{(0)}=\right.$ $\left.0, x_{\alpha}^{\left(N_{\alpha}\right)}=l_{\alpha}, h_{\alpha i_{\alpha}}=x_{\alpha}^{\left(i_{\alpha}\right)}-x_{\alpha}^{\left(i_{\alpha}-1\right)}, i_{\alpha}=\overline{1, N_{\alpha}}\right\}, \alpha=1,2$, as well as the non-uniform grid w.r.t. $x_{1}$ and $x_{2}$ in the domain $\bar{\Omega}=\bar{\Omega}_{1} \cup \bar{\Omega}_{2}: \widehat{\omega}=\widehat{\omega}_{1} \times \widehat{\omega}_{2}$. It is clear that one can always construct grid $\widehat{\omega}_{1}$ on $\left[0, l_{1}\right]$ so that the point $x_{1}=\xi$ is its node.

While solving practical problems, it is reasonable to choose uniform steps $h_{1}^{(1)}$ and $h_{1}^{(2)}$ in domains $\bar{\Omega}_{1}$ and $\bar{\Omega}_{2}$, respectively, and basing on the position of the point $x_{1}=\xi$, the number of nodes should be fixed by the assumption $h_{1}^{(1)} \approx h_{1}^{(2)}$. We let $x_{1}^{\left(i_{1}\right)}-x_{1}^{\left(i_{1}-1\right)}=h_{1}, i_{1}=\overline{1, N_{1}}$ and $x_{2}^{\left(i_{2}\right)}-x_{2}^{\left(i_{2}-1\right)}=h_{2}, i_{2}=\overline{1, N_{2}}$. Value $x_{1}$ at the point $x_{1}=\xi$ is denoted by $x_{\xi}$, and the corresponding index of node is indicated by $N_{1 \xi}, 1<N_{1 \xi}<N_{1}-1$. 
We introduce the grids:

$$
\begin{aligned}
& \bar{\omega}_{1}^{(1)}=\left\{x_{1}^{\left(i_{1}\right)}=i_{1} h_{1} \in[0, \xi]: i_{1}=\overline{0, N_{1 \xi}}, N_{1 \xi} h_{1}=\xi\right\}, \\
& \bar{\omega}_{1}^{(2)}=\left\{x_{1}^{\left(i_{1}\right)}=i_{1} h_{1} \in\left[\xi, l_{1}\right]: i_{1}=\overline{N_{1 \xi}, N_{1}}, N_{1 \xi} h_{1}=l_{1}\right\}, \\
& \omega_{1}^{(1)}=\bar{\omega}_{1}^{(1)} \backslash\left\{x_{1}=0, x_{1}=\xi\right\}, \quad \omega_{1}^{(2)}=\bar{\omega}_{1}^{(2)} \backslash\left\{x_{1}=\xi, x_{1}=l_{1}\right\} ; \\
& \bar{\omega}_{2}=\left\{x_{2}^{\left(i_{2}\right)}=i_{2} h_{2} \in\left[0, l_{2}\right]: i_{2}=\overline{0, N_{2}}, N_{2} h_{2}=l_{2}\right\}, \quad \omega_{2}=\bar{\omega}_{2} \backslash\left\{x_{2}=0, x_{2}=l_{2}\right\} ; \\
& \bar{\omega}_{1}=\bar{\omega}_{1}^{(1)} \cup \bar{\omega}_{1}^{(2)} ; \quad \omega_{1}=\omega_{1}^{(1)} \cup \omega_{1}^{(2)} ; \quad \bar{\omega}^{(1)}=\bar{\omega}_{1}^{(1)} \times \bar{\omega}_{2} ; \\
& \bar{\omega}^{(2)}=\bar{\omega}_{1}^{(2)} \times \bar{\omega}_{2} ; \quad \omega^{(1)}=\omega_{1}^{(1)} \times \omega_{2} ; \quad \omega^{(2)}=\omega_{1}^{(2)} \times \omega_{2} ; \\
& \bar{\omega} \equiv \bar{\omega}^{(1,2)}=\bar{\omega}^{(1)} \cup \bar{\omega}^{(2)}=\left(\bar{\omega}_{1}^{(1)} \cup \bar{\omega}_{1}^{(2)}\right) \times \bar{\omega}_{2} \\
& =\left\{x_{1}^{\left(i_{1}\right)}=i_{1} h_{1}, i_{1}=\overline{0, N_{1}}, N_{1 \xi} h_{1}=\xi,\left(N_{1}-N_{1 \xi}\right) h_{1}=l_{1}-\xi, 1<N_{1 \xi}<N_{1}-1\right\} \times \bar{\omega}_{2}, \\
& \omega \equiv \omega^{(1,2)}=\omega^{(1)} \times \omega^{(2)} ; \quad \omega_{1}^{(1)+}=\bar{\omega}_{1}^{(1)} \cap(0, \xi], \quad \omega_{1}^{(1)-}=\bar{\omega}_{1}^{(1)} \cap[0, \xi), \\
& \omega_{1}^{(2)-}=\bar{\omega}_{1}^{(2)} \cap\left[\xi, l_{1}\right), \quad \omega^{(1)(+1)}=\omega_{1}^{(1)+} \times \bar{\omega}_{2} ; \\
& \gamma_{S}=\left\{x_{1}=\xi, x_{2}=h_{2}, 2 h_{2}, \ldots,\left(N_{2}-1\right) h_{2}\right\}=\left\{x_{1}=\xi, x_{2}^{\left(i_{2}\right)}=i_{2} h_{2}, i_{2}=\overline{1, N_{2}-1}\right\} ; \\
& \gamma^{(k)}=\partial \omega^{(k)} \backslash \gamma_{S} ; \quad \omega_{1}^{(1)+} \times \omega_{2}=\omega^{(1)} \cup \gamma_{S}=\bar{\omega}^{(1)} \backslash \gamma^{(1)} ;
\end{aligned}
$$

and $\partial \omega^{(k)}=\bar{\omega}^{(k)} \backslash \omega^{(k)}$ is the set of boundary nodes of grids $\bar{\omega}^{(k)}, k=1,2$. For studying convergence of difference approximations we shall make use of scalar products, norms, and semi-norms of grid functions defined on various grids. The set of grid functions $y_{1}(x)$ defined on the grid $\bar{\omega}^{(1)}=\bar{\omega}_{1}^{(1)} \times \bar{\omega}_{2} \subset \bar{\Omega}_{1} \equiv \bar{\Omega}^{-}$is denoted by $H_{h}^{(1)}\left(\bar{\omega}^{(1)}\right)$, while the set of grid functions $y_{2}(x)$ defined on the grid $\bar{\omega}^{(2)}=\bar{\omega}_{1}^{(2)} \times \bar{\omega}_{2} \subset \bar{\Omega}_{2} \equiv \bar{\Omega}^{+}$is indicated by $H_{h}^{(2)}\left(\bar{\omega}^{(2)}\right)$. Set $H_{h}^{(k)}\left(\bar{\omega}^{(k)}\right)$, $k=1,2$, equipped by the scalar product and norm

$$
\left(y_{k}, \nu_{k}\right)_{L_{2}\left(\bar{\omega}^{(k)}\right)}=\sum_{\bar{\omega}^{(k)}} y_{k}(x) \nu_{k}(x) \hbar_{1} \hbar_{2}, \quad\left\|y_{k}\right\|_{L_{2}\left(\bar{\omega}^{(k)}\right)}=\left(y_{k}, y_{k}\right)_{L_{2}\left(\bar{\omega}^{(k)}\right)}^{1 / 2}
$$

is denoted by $L_{2}\left(\bar{\omega}^{(k)}\right), k=1,2$. Here $\hbar_{1}=\hbar_{1}\left(x_{1}\right)$ is the mean step of grid $\bar{\omega}_{1}^{(1)}$ and $\bar{\omega}_{1}^{(2)}$, and $\hbar_{2}=\hbar_{2}\left(x_{2}\right)$ is the mean step of grid $\bar{\omega}_{2}$, 6]. By $W_{2}^{1}\left(\bar{\omega}^{(1)}\right)$ and $W_{2}^{1}\left(\bar{\omega}^{(2)}\right)$ we denote the spaces of grid functions defined on grids $\bar{\omega}^{(1)}$ and $\bar{\omega}^{(2)}$, respectively, with the scalar products and norms:

$$
\begin{gathered}
\left(y_{k}, \nu_{k}\right)_{W_{2}^{1}\left(\bar{\omega}^{(k)}\right)}=\sum_{\omega_{1}^{(k)+} \times \bar{\omega}_{2}} y_{k \bar{x}_{1}} \nu_{k \bar{x}_{1}} h_{1} \hbar_{2}+\sum_{\bar{\omega}_{1}^{(k)} \times \omega_{2}^{+}} y_{k \bar{x}_{2}} \nu_{k \bar{x}_{2}} \hbar_{1} h_{2}+\left(y_{k}, \nu_{k}\right)_{L_{2}\left(\bar{\omega}^{(k)}\right)}, \\
\left\|y_{k}\right\|_{W_{2}^{1}\left(\bar{\omega}^{(k)}\right)}^{2}=\left\|\nabla y_{k}\right\|^{2}+\left\|y_{k}\right\|_{L_{2}\left(\bar{\omega}^{(k)}\right)}^{2}, \quad k=1,2,
\end{gathered}
$$

where

$$
\left\|\nabla y_{k}\right\|^{2}=\sum_{\omega_{1}^{(k)+} \times \bar{\omega}_{2}} y_{k \bar{x}_{1}}^{2} h_{1} \hbar_{2}+\sum_{\bar{\omega}_{1}^{(k)} \times \omega_{2}^{+}} y_{k \bar{x}_{2}}^{2} \hbar_{1} h_{2}, \quad k=1,2 .
$$

We introduce space $V\left(\bar{\omega}^{(1,2)}\right)$ of pairs of grid functions $y(x)=\left(y_{1}(x), y_{2}(x)\right)$ by the identity $V\left(\bar{\omega}^{(1,2)}\right)=\left\{y(x)=\left(y_{1}(x), y_{2}(x)\right) \in W_{2}^{1}\left(\bar{\omega}^{(1)}\right) \times W_{2}^{1}\left(\bar{\omega}^{(2)}\right)\right\}$. Being equipped with the scalar product and norm

$$
(y, \nu)_{V\left(\bar{\omega}^{(1,2)}\right)}=\sum_{k=1}^{2}\left(y_{k}, \nu_{k}\right)_{W_{2}^{1}\left(\bar{\omega}^{(k)}\right)}, \quad\|y\|_{V\left(\bar{\omega}^{(1,2)}\right)}^{2}=\sum_{k=1}^{2}\left\|y_{k}\right\|_{W_{2}^{1}\left(\bar{\omega}^{(k)}\right)}^{2},
$$

$V\left(\bar{\omega}^{(1,2)}\right)$ is a Hilbert space.

We define grid analogues of scalar products for the traces of grid functions $y_{k}(x)$ and $\nu_{k}(x)$, $x \in \bar{\omega}^{(k)}$, on boundaries $\partial \omega^{(k)}$ of grids $\bar{\omega}^{(k)}, k=1,2$, by the formulae $\left(y_{k}, \nu_{k}\right)_{L_{2}\left(\partial \omega^{(k)}\right)}=$ 
$\sum_{x \in \partial \omega^{(k)}} y_{k}(x) \nu_{k}(x) \tau_{k}(x), k=1,2$, as well as grid analogues of the norms in $L_{2}\left(\partial \omega^{(k)}\right)$ generated by the scalar products

$$
\begin{aligned}
& \left\|y_{k}\right\|_{L_{2}\left(\partial \omega^{(k)}\right)}^{2}=\left(y_{k}, y_{k}\right)_{L_{2}\left(\partial \omega^{(k)}\right)}=\sum_{\partial \omega^{(k)}} y_{k}^{2}(x) \tau_{k}(x), \quad k=1,2, \\
& \tau_{1}(x)= \begin{cases}h_{1}\left(x_{1}\right), & x_{1} \in \omega_{1}^{(1)}, \quad x_{2}=0, l_{2} ; \\
h_{2}\left(x_{2}\right), & x_{2} \in \omega_{2}, x_{1}=0, \xi ; \\
\frac{h_{1}\left(x_{1}\right)+h_{2}\left(x_{2}\right)}{2}, & x \in \gamma^{(1)},\end{cases} \\
& \tau_{2}(x)= \begin{cases}h_{1}\left(x_{1}\right), & x_{1} \in \omega_{1}^{(1)}, \quad x_{2}=0, l_{2} ; \\
h_{2}\left(x_{2}\right), & x_{2} \in \omega_{2}, \quad x_{1}=\xi, l_{1} ; \\
\frac{h_{1}\left(x_{1}\right)+h_{2}\left(x_{2}\right)}{2}, & x \in \gamma^{(2)},\end{cases}
\end{aligned}
$$

and $q^{\prime}(k)$ is the set of the corner points of rectangle $\Omega_{k}, k=1,2$. For instance, written in all the details, the grid analogue of the norm in $L_{2}\left(\partial \omega^{(1)}\right)$ is defined by the identity

$$
\left\|y_{1}\right\|_{L_{2}\left(\partial \omega^{(1)}\right)}^{2}=\sum_{x_{2} \in \bar{\omega}_{2}}\left[y_{1}^{2}\left(0, x_{2}\right)+y_{1}^{2}\left(\xi, x_{2}\right)\right] \hbar_{2}\left(x_{2}\right)+\sum_{x_{1} \in \bar{\omega}_{1}}\left[y_{1}^{2}\left(x_{1}, 0\right)+y_{1}^{2}\left(x_{1}, l_{2}\right)\right] \hbar_{1}\left(x_{1}\right) .
$$

Suppose now that $\stackrel{\circ}{\gamma}^{(k)}=\partial \omega^{(k)} \cap \stackrel{\circ}{\Gamma}_{k} \equiv \gamma^{(k)}=\partial \omega^{(k)} \backslash \gamma_{s}$ is the subset of boundary nodes $\partial \omega^{(k)}$ of grid $\bar{\omega}^{(k)} \subset \bar{\Omega}_{k}, k=1,2$. By $L_{2}\left(\bar{\omega}^{(k)} ; \gamma^{(k)}\right)$ we denote the normed subspace of space of grid functions $L_{2}\left(\bar{\omega}^{(k)}\right)$ vanishing on $\gamma^{(k)}, k=1,2$, with the norms

$$
\begin{aligned}
\left\|y_{k}\right\|_{L_{2}\left(\bar{\omega}^{(k)} ; \gamma^{(k)}\right)}^{2} & =\sum_{x \in \omega^{(k)}} y_{k}^{2}(x) h_{1} h_{2}+\frac{1}{2} \sum_{x \in \gamma_{S}} y_{k}^{2}(x) h_{1} h_{2} \\
& =\sum_{x \in \omega^{(k)}} y_{k}^{2}(x) h_{1} h_{2}+\frac{1}{2} \sum_{x_{2} \in \omega_{2}} y_{k}^{2}\left(\xi, x_{2}\right) h_{1} h_{2}, \quad k=1,2,
\end{aligned}
$$

induced by the scalar products

$$
\left(y_{k}, v_{k}\right)_{L_{2}\left(\bar{\omega}^{(k)} ; \gamma^{(k)}\right)}=\sum_{x \in \omega^{(k)}} y_{k}(x) v_{k}(x) h_{1} h_{2}+\frac{1}{2} \sum_{x \in \gamma_{S}} y_{k}(x) v_{k}(x) h_{1} h_{2}, \quad k=1,2 .
$$

It is easy to see that

$$
\left(y_{1}, v_{1}\right)_{L_{2}\left(\bar{\omega}^{(1)} ; \gamma^{(1)}\right)}=\left(y_{1}, v_{1}\right)_{L_{2}\left(\omega_{1}^{(1)+} \times \omega_{2}\right)}, \quad\left(y_{2}, v_{2}\right)_{L_{2}\left(\bar{\omega}^{(2)} ; \gamma^{(2)}\right)}=\left(y_{2}, v_{2}\right)_{L_{2}\left(\omega_{2}^{(2)-} \times \omega_{2}\right)} .
$$

By $W_{2}^{1}\left(\bar{\omega}^{(k)} ; \gamma^{(k)}\right)$ we indicate the subspace of space of grid functions $W_{2}^{1}\left(\bar{\omega}^{(k)}\right)$ vanishing on $\gamma^{(k)}, k=1,2$.

We introduce spaces $\stackrel{\circ}{H}_{\gamma^{(1)} \gamma^{(2)}}\left(\bar{\omega}^{(1,2)}\right)$ and $\stackrel{\circ}{V}_{\gamma^{(1)} \gamma^{(2)}}\left(\bar{\omega}^{(1,2)}\right)$ of pairs of grid functions $y(x)=$ $\left(y_{1}(x), y_{2}(x)\right)$ :

$$
\begin{aligned}
& \stackrel{\circ}{H}_{\gamma^{(1)} \gamma^{(2)}}\left(\bar{\omega}^{(1,2)}\right)=\left\{y(x)=\left(y_{1}(x), y_{2}(x)\right) \in L_{2}\left(\bar{\omega}^{(1)} ; \gamma^{(1)}\right) \times L_{2}\left(\bar{\omega}^{(2)} ; \gamma^{(2)}\right)\right\}, \\
& \stackrel{\circ}{V}_{\gamma^{(1)} \gamma^{(2)}}\left(\bar{\omega}^{(1,2)}\right)=\left\{y(x)=\left(y_{1}(x), y_{2}(x)\right) \in W_{2}^{1}\left(\bar{\omega}^{(1)} ; \gamma^{(1)}\right) \times W_{2}^{1}\left(\bar{\omega}^{(2)} ; \gamma^{(2)}\right)\right\}
\end{aligned}
$$

with the norms

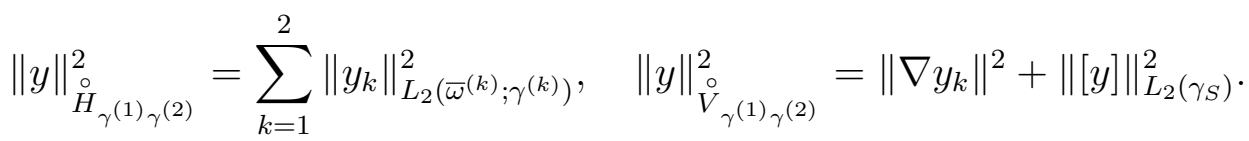


By $e_{1}^{(1)}\left(x_{1}\right)$ we denote elementary sells of the segment $[0, \xi]$ :

$$
\begin{aligned}
& e_{1}^{(1)}\left(x_{1}\right)=\left\{r_{1}: x_{1}-0.5 h_{1} \leqslant r_{1} \leqslant x_{1}+0.5 h_{1}\right\}, \quad x_{1} \in \omega_{1}^{(1)} \subset[0, \xi], \\
& e_{1}^{(1)}(0)=\left\{r_{1}: 0 \leqslant r_{1} \leqslant 0.5 h_{1}\right\}, \quad e_{1}^{(1)}(\xi)=\left\{r_{1}: \xi-0.5 h_{1} \leqslant r_{1} \leqslant \xi\right\}
\end{aligned}
$$

while $e_{1}^{(2)}\left(x_{1}\right)$ stands for the elementary sells of the segment $\left[\xi, l_{1}\right]$ :

$$
\begin{aligned}
& e_{1}^{(2)}\left(x_{1}\right)=\left\{r_{1}: x_{1}-0.5 h_{1} \leqslant r_{1} \leqslant x_{1}+0.5 h_{1}\right\}, \quad x_{1} \in \omega_{1}^{(2)} \subset\left[\xi, l_{1}\right], \\
& e_{1}^{(2)}(\xi)=\left\{r_{1}: \xi \leqslant r_{1} \leqslant \xi+0.5 h_{1}\right\}, \quad e_{1}^{(2)}\left(l_{1}\right)=\left\{r_{1}: l_{1}-0.5 h_{1} \leqslant r_{1} \leqslant l_{1}\right\} .
\end{aligned}
$$

We also introduce elementary sells of the segment $\left[0, l_{2}\right]$ :

$$
\begin{aligned}
& e_{2}\left(x_{2}\right)=\left\{r_{2}: x_{2}-0.5 h_{2} \leqslant r_{2} \leqslant x_{2}+0.5 h_{2}\right\}, \quad x_{2} \in \omega_{2} \subset\left[0, l_{2}\right], \\
& e_{2}(0)=\left\{r_{2}: 0 \leqslant r_{2} \leqslant 0.5 h_{2}\right\}, \quad e_{2}\left(l_{2}\right)=\left\{r_{2}: l_{2}-0.5 h_{2} \leqslant r_{2} \leqslant l_{2}\right\}
\end{aligned}
$$

$\operatorname{By} e^{(1)}(x) \equiv e^{(1)}\left(x_{1}, x_{2}\right)=e_{1}^{(1)}\left(x_{1}\right) \times e_{2}\left(x_{2}\right), x \in \bar{\omega}^{(1)}=\bar{\omega}_{1}^{(1)} \times \bar{\omega}_{2} \subset \bar{\Omega}_{1}$ we denote elementary sells of domain $\bar{\Omega}_{1}$, while $e^{(2)}(x) \equiv e^{(2)}\left(x_{1}, x_{2}\right)=e_{1}^{(2)}\left(x_{1}\right) \times e_{2}\left(x_{2}\right), x \in \bar{\omega}^{(2)}=\bar{\omega}_{1}^{(2)} \times \bar{\omega}_{2} \subset \bar{\Omega}_{2}$ are the elementary sells of domain $\bar{\Omega}_{2}$. Let $v(x)=v_{1}(x), x \in \bar{\Omega}_{1}$. For functions $v_{1}(x), x \in \bar{\Omega}_{1}$ we define Steklov averaging operators $S^{x_{\alpha}}$ w.r.t. variables $x_{\alpha}, \alpha=1,2$ :

$$
\begin{aligned}
& S^{x_{1}} v_{1}(x)=\frac{1}{\hbar_{1}} \int_{e_{1}^{(1)}\left(x_{1}\right)} v_{1}\left(r_{1}, x_{2}\right) d r_{1}, \quad x_{1} \in \bar{\omega}_{1}^{(1)}, \quad \hbar_{1}=\hbar_{1}\left(x_{1}\right)= \begin{cases}h_{1}, & x_{1} \in \omega_{1}^{(1)} \\
0.5 h_{1}, & x_{1}=0, \xi\end{cases} \\
& S^{x_{2}} v_{1}(x)=\frac{1}{\hbar_{2}} \int_{e_{2}\left(x_{2}\right)} v_{1}\left(x_{1}, r_{2}\right) d r_{2}, \quad x_{2} \in \bar{\omega}_{2}, \quad \hbar_{2}=\hbar_{2}\left(x_{2}\right)= \begin{cases}h_{2}, & x_{2} \in \omega_{2}, \\
0.5 h_{2}, & x_{2}=0, l_{2} .\end{cases}
\end{aligned}
$$

By means of one-dimensional operators $S^{x_{\alpha}}$ acting in the direction of $x_{\alpha}, \alpha=1,2$, we define the averaging operator $S^{x}=S^{x_{1}} S^{x_{2}}$ as the product of one-dimensional averaging operators. In the same way we define Steklov averaging operators for functions $v(x)=v_{2}(x), x \in \bar{\Omega}_{2}$. In what follows by $H_{h}^{(1)}\left(\omega^{(1)} \cup \gamma_{S}\right) \equiv L_{2}\left(\omega^{(1)} \cup \gamma_{S}\right)$ we denote the set of grid functions $v_{1 h}(x)$, $x \in \omega^{(1)} \cup \gamma_{S}$ defined on the grid $\omega^{(1)} \cup \gamma_{S}$ with the scalar product and norm

$$
\begin{gathered}
\left(v_{1 h}, \tilde{v}_{1 h}\right)_{H_{h}^{(1)}\left(\omega^{(1)} \cup \gamma_{S}\right)}=\sum_{x \in \omega^{(k)}} v_{1 h}(x) \tilde{v}_{1 h}(x) h_{1} h_{2}+\frac{1}{2} \sum_{x \in \gamma_{S}} v_{1 h}(x) \tilde{v}_{1 h}(x) h_{1} h_{2}, \\
\left\|v_{1 h}(x)\right\|_{H_{h}^{(1)}\left(\omega^{(1)} \cup \gamma_{S}\right)}^{2}=\left(v_{1 h}, v_{1 h}\right)_{H_{h}^{(1)}\left(\omega^{(1)} \cup \gamma_{S}\right)} .
\end{gathered}
$$

In the same way we define the space of grid functions $H_{h}^{(2)}\left(\omega^{(2)} \cup \gamma_{S}\right) \equiv L_{2}\left(\omega^{(2)} \cup \gamma_{S}\right)$.

To optimal control problem (1)-(3) we associate the following difference approximations: to minimize the grid functional

$$
J_{h}\left(\Phi_{h}\right)=\sum_{x \in \bar{\omega}^{(1)}}\left|y\left(x, \Phi_{h}\right)-u_{0 h}^{(1)}(x)\right|^{2} \hbar_{1} \hbar_{2}=\left\|y\left(x, \Phi_{h}\right)-u_{0 h}^{(1)}(x)\right\|_{L_{2}\left(\bar{\omega}^{(1)}\right)}^{2}
$$

under the conditions that grid function $y(x) \equiv y\left(x, \Phi_{h}\right)=\left(y_{1}\left(x, \Phi_{h}\right), y_{2}\left(x, \Phi_{h}\right)\right) \in \stackrel{\circ}{V}_{\gamma^{(1)} \gamma^{(2)}}$ $\left(\bar{\omega}^{(1,2)}\right)$ called the solution to the difference boundary value problems (difference scheme) for 
problem (1) satisfies the summation identity

$$
\begin{aligned}
& Q_{h}(y, v)=\left\{\sum_{\omega_{1}^{(1)+}} \sum_{\omega_{2}} a_{1 h}^{(1)} y_{1 \bar{x}_{1}} v_{1 \bar{x}_{1}} h_{1} h_{2}+\left(\sum_{\omega_{1}^{(1)}} \sum_{\omega_{2}^{+}} a_{2 h}^{(1)} y_{1 \bar{x}_{2}} v_{1 \bar{x}_{2}} h_{1} h_{2}\right.\right. \\
& \left.\left.+\frac{1}{2} \sum_{\omega_{2}^{+}} a_{2 h}^{(1)}\left(\xi, x_{2}\right) y_{1 \bar{x}_{2}}\left(\xi, x_{2}\right) v_{1 \bar{x}_{2}}\left(\xi, x_{2}\right) h_{1} h_{2}\right)\right\}+\left\{\sum_{\omega_{1}^{(2)+}} \sum_{\omega_{2}} a_{1 h}^{(2)} y_{2 \bar{x}_{1}} v_{2 \bar{x}_{1}} h_{1} h_{2}\right. \\
& \left.+\left(\sum_{\omega_{1}^{(2)}} \sum_{\omega_{2}^{+}} a_{2 h}^{(2)} y_{2 \bar{x}_{2}} v_{2 \bar{x}_{2}} h_{1} h_{2}+\frac{1}{2} \sum_{\omega_{2}^{+}} a_{2 h}^{(2)}\left(\xi, x_{2}\right) y_{2 \bar{x}_{2}}\left(\xi, x_{2}\right) v_{2 \bar{x}_{2}}\left(\xi, x_{2}\right) h_{1} h_{2}\right)\right\} \\
& +\sum_{\omega_{2}} \Phi_{h}(x)\left[y\left(\xi, x_{2}\right)\right]\left[v\left(\xi, x_{2}\right)\right] h_{2}+\left\{\left(\sum_{\omega(1)} d_{1 h}(x) q_{1}\left(y_{1}(x)\right) v_{1}(x) h_{1} h_{2}\right.\right. \\
& \left.+\frac{1}{2} \sum_{\omega_{2}} d_{1 h}\left(\xi, x_{2}\right) q_{1}\left(y_{1}\left(\xi, x_{2}\right)\right) v_{1}\left(\xi, x_{2}\right) h_{1} h_{2}\right)+\left(\sum_{\omega(2)} d_{2 h}(x) q_{2}\left(y_{2}(x)\right) v_{2}(x) h_{1} h_{2}\right. \\
& \left.\left.+\frac{1}{2} \sum_{\omega_{2}} d_{2 h}\left(\xi, x_{2}\right) q_{2}\left(y_{2}\left(\xi, x_{2}\right)\right) v_{2}\left(\xi, x_{2}\right) h_{1} h_{2}\right)\right\} \\
& =\left\{\left(\sum_{\omega(1)} f_{1 h}(x) v_{1}(x) h_{1} h_{2}+\frac{1}{2} \sum_{\omega_{2}} f_{1 h}\left(\xi, x_{2}\right) v_{1}\left(\xi, x_{2}\right) h_{1} h_{2}\right)+\right. \\
& \left.+\left(\sum_{\omega(2)} f_{2 h}(x) v_{2}(x) h_{1} h_{2}+\frac{1}{2} \sum_{\omega_{2}} f_{2 h}\left(\xi, x_{2}\right) v_{2}\left(\xi, x_{2}\right) h_{1} h_{2}\right)\right\}=l_{h}(v),
\end{aligned}
$$

for each grid function $v(x)=\left(v_{1}\left(x, \Phi_{h}\right), v_{2}\left(x, \Phi_{h}\right)\right) \in \stackrel{\circ}{V}_{\gamma^{(1)} \gamma^{(2)}}\left(\bar{\omega}^{(1,2)}\right)$, while grid controls $\Phi_{h}(x)$, $x \in \gamma_{S}$ are so that

$$
\Phi_{h}(x) \in U_{h}=\left\{\Phi_{h} \in L_{2}\left(\gamma_{S}\right)=H_{h}: 0<g_{0} \leqslant \Phi_{h}(x) \leqslant \bar{g}_{0}, x \in \gamma_{S}\right\},
$$

where $L_{2}\left(\gamma_{S}\right)=H_{h}$ is the space of grid controls $\Phi_{h}$ defined on grid $\gamma_{S} \subset S$ with the scalar product and norm

$$
\left(\Phi_{h}, \tilde{\Phi}_{h}\right)_{L_{2}\left(\gamma_{S}\right)}=\sum_{x \in \gamma_{S}} h_{2} \Phi_{h}(x) \tilde{\Phi}_{h}(x), \quad\left\|\Phi_{h}(x)\right\|_{L_{2}\left(\gamma_{S}\right)}^{2}=\left(\Phi_{h}, \Phi_{h}\right)_{L_{2}\left(\gamma_{S}\right)} .
$$

By $a_{\alpha h}^{(1)}(x), a_{\alpha h}^{(2)}(x), d_{\alpha h}(x), \alpha=1,2, f_{1 h}(x), f_{2 h}(x), u_{0 h}^{(1)}(x)$ we denote grid approximations for functions $k_{\alpha}^{(1)}(r), k_{\alpha}^{(2)}(r), d_{\alpha}(r), \alpha=1,2, f_{1}(r), f_{2}(r), u_{0}^{(1)}(r)$ defined by Steklov averages:

$$
\begin{aligned}
& a_{1 h}^{(\alpha)}\left(x_{1}, x_{2}\right)=\frac{1}{h_{2}} \int_{e_{2}\left(x_{2}\right)} k_{1}^{(\alpha)}\left(x_{1}-0.5 h_{1}, r_{2}\right) d r_{2}, \quad x \in \omega_{1}^{(\alpha)+} \times \omega_{2}, \quad \alpha=1,2 ; \\
& a_{2 h}^{(\alpha)}\left(x_{1}, x_{2}\right)=\frac{1}{h_{1}} \int_{e_{1}^{(\alpha)}\left(x_{1}\right)} k_{2}^{(\alpha)}\left(r_{1}, x_{2}-0.5 h_{2}\right) d r_{1}, \quad x \in \omega_{1}^{(\alpha)} \times \omega_{2}^{+}, \quad \alpha=1,2 ; \\
& a_{2 h}^{(1)}\left(\xi, x_{2}\right)=\frac{2}{h_{1}} \int_{\xi-0.5 h_{1}}^{\xi} k_{2}^{(1)}\left(r_{1}, x_{2}-0.5 h_{2}\right) d r_{1}, \quad x_{2} \in \omega_{2}^{+} ;
\end{aligned}
$$




$$
\begin{aligned}
& a_{2 h}^{(2)}\left(\xi, x_{2}\right)=\frac{2}{h_{1}} \int_{\xi}^{\xi+0.5 h_{1}} k_{2}^{(2)}\left(r_{1}, x_{2}-0.5 h_{2}\right) d r_{1}, \quad x_{2} \in \omega_{2}^{+} ; \\
& d_{\alpha h}(x)=\frac{1}{h_{1} h_{2}} \iint_{e^{\alpha}(x)} d_{\alpha}\left(r_{1}, r_{2}\right) d r_{1} d r_{2}, \quad x \in \omega^{(\alpha)}, \alpha=1,2 ; \\
& d_{1 h}\left(\xi, x_{2}\right)=\frac{2}{h_{1} h_{2}} \int_{\xi-0.5 h_{1}} \int_{e_{2}\left(x_{2}\right)} d_{1}\left(r_{1}, r_{2}\right) d r_{1} d r_{2}, \quad x_{2} \in \omega_{2} ; \\
& d_{2 h}\left(\xi, x_{2}\right)=\frac{2}{h_{1} h_{2}} \int_{\xi}^{\xi+0.5 h_{1}} \int_{e_{2}\left(x_{2}\right)} d_{2}\left(r_{1}, r_{2}\right) d r_{1} d r_{2}, \quad x_{2} \in \omega_{2} ; \\
& f_{\alpha h}(x)=\frac{1}{h_{1} h_{2}} \iint_{e^{(\alpha)}} f_{\alpha}\left(r_{1}, r_{2}\right) d r_{1} d r_{2}, \quad x \in \omega^{(\alpha)}, \quad \alpha=1,2 ; \\
& f_{1 h}\left(\xi, x_{2}\right)=\frac{2}{h_{1} h_{2}} \int_{\xi-0.5 h_{1}}^{\xi} \int_{e_{2}\left(x_{2}\right)} f_{1}\left(r_{1}, r_{2}\right) d r_{1} d r_{2}, \quad x_{2} \in \omega_{2} ; \\
& u_{0 h}^{(1)}(x)=\frac{1}{h_{1} \hbar_{2}} \int_{e^{(1)}(x)} \int_{\xi} \int_{h_{1}}^{(1)}\left(r_{1}, r_{2}\right) d r_{1} d r_{2}, \quad x \in \bar{\omega}^{(1)}=\bar{\omega}_{1}^{(1)} \times \bar{\omega}_{2} . \\
& f_{2 h}\left(\xi, x_{2}\left(r_{1}, r_{2}\right) d r_{1} d r_{2}, \quad x_{2} \in \omega_{2} ;\right.
\end{aligned}
$$

Theorem 2. Problem on solving difference scheme $(12)$ for each fixed control $\Phi_{h} \in U_{h}$ is equivalent to solving operator equation $A_{h} y=F_{h}$, where difference operator $A_{h}$ acting from $\stackrel{\circ}{V}_{\gamma^{(1)} \gamma^{(2)}}\left(\bar{\omega}^{(1,2)}\right)$ into $\stackrel{\circ}{V}_{\gamma^{(1)} \gamma^{(2)}}\left(\bar{\omega}^{(1,2)}\right)$ and grid function $F_{h} \in \stackrel{\circ}{V}_{\gamma^{(1)} \gamma^{(2)}}\left(\bar{\omega}^{(1,2)}\right)$ are defined by the identities

$$
\left(A_{h} y, v\right)_{{\stackrel{\circ}{\gamma^{(1)} \gamma}(2)}_{\left(\bar{\omega}^{(1,2)}\right)}}=Q_{h}(y, v), \quad\left(F_{h}, v\right)_{{\stackrel{\circ}{\gamma^{(1)} \gamma^{(2)}}\left(\bar{\omega}^{(1,2)}\right)}}=l_{h}(v), \forall y, v \in{\stackrel{\circ}{V^{(1)} \gamma^{(2)}}} .
$$

Problem (difference scheme) (12) is uniquely solvable for each grid control $\Phi_{h} \in U_{h}$. At that, the apriori estimate

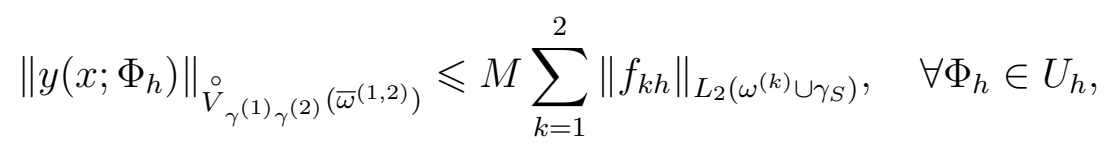

holds true.

Proof. Employing the restrictions for the initial data in boundary value problem (1), CauchySchwarz and Hölder inequalities, difference analogues of the embedding theorems, one can make sure that for each fixed $y \in \stackrel{\circ}{V}_{\gamma^{(1)} \gamma^{(2)}}\left(\bar{\omega}^{(1,2)}\right)$ and for each $\Phi_{h} \in U_{h}$, form $Q_{h}(y, v)$ and $l_{h}(v)$ determine linear bounded functionals in the space of grid functions $\stackrel{\circ}{V}_{\gamma^{(1)} \gamma^{(2)}}\left(\bar{\omega}^{(1,2)}\right)$ and thus, they are uniquely represented by identities (14). Together with $(12)$ and by the arbitrariness of $v$ it implies that identity (12) defines operator equation $A_{h} y=F_{h}$, i.e., the difference scheme. Moreover, one can make sure that operator $A_{h}$ of difference scheme 12 preserves the main properties of differential operator in original problem (1), namely, its strong monotonicity and 
Lipschitz continuity. Therefore, the hypothesis of Browder theorem [21] is obeyed, and thus, equation $A_{h} y=F_{h}$ is uniquely solvable. Estimate 15 follows from the coercitivity of operator $A_{h}$. The proof is complete.

Problem (12) is a grid analogue of original problem for state (1) with discontinuous coefficients and solution (state).

Let us find a relatinop between solution $u(r ; g)$ to direct problem (1) with discontinuous coefficients and solution $y\left(x, \Phi_{h}\right)=\left(y_{1}\left(x ; \Phi_{h}\right), y_{2}\left(x ; \Phi_{h}\right)\right)$ to approximating difference problem for state (12) as $h \rightarrow 0$ for each fixed control $g \in U$ and $\Phi_{h} \in U_{h}$, where $U$ and $U_{h}$ are the sets of admissible controls in optimal control problems (1)-(3) and (11)-(13), respectively. Let $u(r ; g)=\left(u_{1}(r ; g), u_{2}(r ; g)\right) \in \widehat{\circ}_{\Gamma_{1} \Gamma_{2}}\left(\Omega^{(1,2)}\right)$ be the solution to direct problem (1) associated an admissible control $g \in U$, and $y\left(x, \Phi_{h}\right)=\left(y_{1}\left(x ; \Phi_{h}\right), y_{2}\left(x ; \Phi_{h}\right)\right) \in \stackrel{\circ}{V}_{\gamma^{(1)} \gamma^{(2)}}\left(\bar{\omega}^{(1,2)}\right)$ be the solution to problem (12) associated with an admissible control $\Phi_{h} \in U_{h}$. we denote byz $(x) \equiv$ $z\left(x ; g, \Phi_{h}\right)=\left(z_{1}\left(x ; g, \Phi_{h}\right), z_{2}\left(x ; g, \Phi_{h}\right)\right)=\left(y_{1}\left(x ; \Phi_{h}\right)-u_{1}(r ; g), y_{2}\left(x ; \Phi_{h}\right)-u_{2}(r ; g)\right)$ the error of the method w.r.t. state.

To find error $z(x)$ of difference problem $(12)$, we obviously obtain the equation $A_{h} y-A_{h} u=$ $\psi_{h}$, where grid function $\psi_{h}$ is the error of approximation by difference scheme (12) is determined by the identity

$$
\left(\psi_{h}, v\right)_{{\stackrel{\circ}{\gamma^{1} \gamma^{2}}}^{\left(\bar{\omega}^{(1,2)}\right)}}=\left(F_{h}-A_{h} u, v\right)_{{\stackrel{\circ}{\gamma^{1} \gamma^{2}}}^{\left(\bar{\omega}^{(1,2)}\right)}}=l_{h}(v)-Q_{h}(u, v), \quad \forall v \in{\stackrel{\circ}{\gamma^{1} \gamma^{2}}}\left(\bar{\omega}^{(1,2)}\right) .
$$

The next theorem provides an apriori estimate for the error of the method.

Theorem 3. Let $g \in U$ and $\Phi_{h} \in U_{h}$ be arbitrary control, $u(r ; g)$ and $y\left(x, \Phi_{h}\right)$ be the associated solutions to state problems in extremum problems (1) - (3) and (11) - (13). Then for each $h>0$ the estimate for the rate of convergence of grid method w.r.t. state

$$
\begin{aligned}
& \left\|y\left(x ; \Phi_{h}\right)-u(x ; g)\right\|_{{\stackrel{\circ}{\gamma^{1} \gamma^{2}}}^{\left(\bar{\omega}^{(1,2)}\right)}} \leqslant C\left\{| h | \left[\sum _ { \alpha = 1 } ^ { 2 } \left(\left\|k_{1}^{(\alpha)}\right\|_{L_{\infty}\left(\Omega_{\alpha}\right)}+\right.\right.\right. \\
& \left.+\left\|k_{2}^{(\alpha)}\right\|_{L_{\infty}\left(\Omega_{\alpha}\right)}+L\left\|d_{\alpha}\right\|_{L_{\infty}\left(\Omega_{\alpha}\right)}\right)\left\|u_{\alpha}\right\|_{W_{2}^{2}\left(\Omega_{\alpha}\right)} \\
& \left.\left.+\|\theta\|_{L_{\infty}\left(0, l_{2}\right)} \sum_{\alpha=1}^{2}\left\|u_{\alpha}\right\|_{W_{2}^{2}\left(\Omega_{\alpha}\right)}\right]+\left\|S^{x_{2}} \theta\left(x_{2}\right)-\Phi_{h}\left(x_{2}\right)\right\|_{L_{\infty}\left(\omega_{2}\right)} \sum_{\alpha=1}^{2}\left\|u_{\alpha}\right\|_{W_{2}^{2}\left(\Omega_{\alpha}\right)}\right\} .
\end{aligned}
$$

holds true for extremum problem (1)-(3).

Proof. We employ difference Green formula and formula for difference integration by parts and ideas of works [1]-[5], [10]-[13] to rewrite approximation error $\psi_{h}(x)$ by rather cumbersome transformations to the special form:

$$
\begin{aligned}
\left(\psi_{h}, v\right)_{\left.{\stackrel{\circ}{\gamma^{1} \gamma^{2}}}^{(} \bar{\omega}^{(1,2)}\right)}= & -\sum_{\alpha=1}^{2} \sum_{\omega_{1}^{(\alpha)+}} \sum_{\omega_{2}} \eta_{1}^{(\alpha)}(x) v_{\alpha \bar{x}_{1}} h_{1} h_{2}-\sum_{\alpha=1}^{2} \sum_{\omega_{1}^{(\alpha)}} \sum_{\omega_{2}^{+}} \eta_{2}^{(\alpha)}(x) v_{\alpha \bar{x}_{2}} h_{1} h_{2} \\
& -\frac{1}{2} \sum_{\omega_{2}^{+}} \eta_{2}^{(1)}\left(\xi, x_{2}\right) v_{1 \bar{x}_{2}}\left(\xi, x_{2}\right) h_{1} h_{2}-\frac{1}{2} \sum_{\omega_{2}^{+}} \eta_{2}^{(2)}\left(\xi, x_{2}\right) v_{2 \bar{x}_{2}}\left(\xi, x_{2}\right) h_{1} h_{2} \\
& +\sum_{\alpha=1}^{2} \sum_{\omega^{(\alpha)}} \eta_{3}^{(\alpha)}(x) v_{\alpha}(x) h_{1} h_{2}+\frac{1}{2} \sum_{\omega_{2}} \eta_{3}^{(1)}\left(\xi, x_{2}\right) v_{1}\left(\xi, x_{2}\right) h_{1} h_{2} \\
& +\frac{1}{2} \sum_{\omega_{2}} \eta_{3}^{(2)}\left(\xi, x_{2}\right) v_{2}\left(\xi, x_{2}\right) h_{1} h_{2}-\sum_{\omega_{2}} \eta_{4}\left(x_{2}\right)\left[v\left(\xi, x_{2}\right)\right] \cdot h_{2},
\end{aligned}
$$


where

$$
\begin{gathered}
\eta_{1}^{(\alpha)}(x)=a_{1 h}^{(\alpha)}(x) u_{\alpha \bar{x}_{1}}(x)-\frac{1}{h_{2}} \int_{e_{2}\left(x_{2}\right)} k_{1}^{(\alpha)}\left(x_{1}-0.5 h_{1}, r_{2}\right) \\
\cdot \frac{\partial u_{\alpha}\left(x_{1}-0.5 h_{1}, r_{2}\right)}{\partial r_{1}} d r_{2}, \quad x \in \omega_{1}^{(\alpha)+} \times \omega_{2}, \quad \alpha=1,2 ; \\
\eta_{2}^{(\alpha)}(x)=a_{2 h}^{(\alpha)}(x) u_{\alpha \bar{x}_{2}}(x)-\frac{1}{h_{1}} \int_{e_{1}^{(\alpha)}\left(x_{1}\right)} k_{2}^{(\alpha)}\left(r_{1}, x_{2}-0.5 h_{2}\right) \\
\cdot \frac{\partial u_{\alpha}\left(r_{1}, x_{2}-0.5 h_{2}\right)}{\partial r_{2}} d r_{1}, \quad x \in \omega_{1}^{(\alpha)} \times \omega_{2}^{+}, \quad \alpha=1,2 ; \\
\eta_{2}^{(1)}\left(\xi, x_{2}\right)=a_{2 h}^{(1)}\left(\xi, x_{2}\right) u_{1 \bar{x}_{2}}\left(\xi, x_{2}\right)-\frac{2}{h_{1}} \int_{\xi-0.5 h_{1}}^{\xi} k_{2}^{(1)}\left(r_{1}, x_{2}-0.5 h_{2}\right) \\
\cdot \frac{\partial u_{1}\left(r_{1}, x_{2}-0.5 h_{2}\right)}{\partial r_{2}} d r_{1}, \quad x_{2} \in \omega_{2}^{+} ;
\end{gathered}
$$

and

$$
\begin{aligned}
& \eta_{2}^{(2)}\left(\xi, x_{2}\right)=a_{2 h}^{(2)}\left(\xi, x_{2}\right) u_{2 \bar{x}_{2}}\left(\xi, x_{2}\right)-\frac{2}{h_{1}} \int_{\xi}^{\xi+0.5 h_{1}} k_{2}^{(2)}\left(r_{1}, x_{2}-0.5 h_{2}\right) \frac{\partial u_{1}\left(r_{1}, x_{2}-0.5 h_{2}\right)}{\partial r_{2}} d r_{1}, \quad x_{2} \in \omega_{2}^{+} ; \\
& \eta_{3}^{(\alpha)}(x)=d_{\alpha h}(x) q_{\alpha}\left(u_{\alpha}(x)\right)-\frac{1}{h_{1} h_{2}} \iint_{e^{(\alpha)}(x)} d_{\alpha}(r) q_{\alpha}\left(u_{\alpha}(r)\right) d r, \quad x \in \omega^{(\alpha)}, \quad \alpha=1,2 ; \\
& \eta_{3}^{(1)}\left(\xi, x_{2}\right)=d_{1 h}\left(\xi, x_{2}\right) q_{1}\left(u_{1}\left(\xi, x_{2}\right)\right)-\frac{2}{h_{1} h_{2}} \int_{\xi-0.5 h_{1}}^{\xi} \int_{e_{2}\left(x_{2}\right)} d_{1}(r) q_{1}\left(u_{1}(r)\right) d r, \quad x_{2} \in \omega_{2} ; \\
& \eta_{3}^{(2)}\left(\xi, x_{2}\right)=d_{2 h}\left(\xi, x_{2}\right) q_{2}\left(u_{2}\left(\xi, x_{2}\right)\right)-\frac{2}{h_{1} h_{2}} \int_{\xi}^{\xi+0.5 h_{1}} \int_{e_{2}\left(x_{2}\right)} d_{2}(r) q_{2}\left(u_{2}(r)\right) d r, \quad x_{2} \in \omega_{2} ; \\
& \eta_{4}\left(x_{2}\right)=\Phi_{h}\left(x_{2}\right)\left[u\left(\xi, x_{2}\right)\right]-\frac{2}{h_{2}} \int_{e_{2}\left(x_{2}\right)} \theta\left(r_{2}\right)\left[u\left(\xi, x_{2}\right)\right] d r_{2}, \quad x_{2} \in \omega_{2} .
\end{aligned}
$$

Taking into consideration the equation for the error $A_{h} y-A_{h} u=\psi_{h}$, representation (16), as well as difference analogues of Sobolev embedding theorems, equivalent norms in space $V_{\gamma^{1} \gamma^{2}\left(\bar{\omega}^{(1,2)}\right)}$ (see above), Cauchy-Schwarz and Hölder inequalities, we obtain the estimate

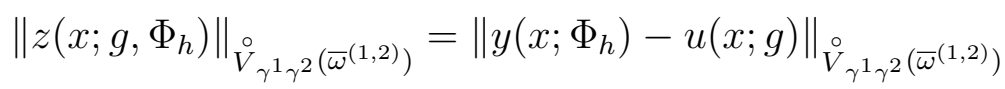

$$
\begin{aligned}
& \leqslant M\left\{\sum _ { \alpha = 1 } ^ { 2 } \left[\left(\sum_{\omega_{1}^{(\alpha)+}} \sum_{\omega_{2}}\left(\eta_{1}^{(\alpha)}(x)\right)^{2} h_{1} h_{2}\right)^{1 / 2}+\left(\sum_{\omega_{1}^{(\alpha)}} \sum_{\omega_{2}^{+}}\left(\eta_{2}^{(\alpha)}(x)\right)^{2} h_{1} h_{2}\right)^{1 / 2}\right.\right. \\
& +\left(\sum_{\omega_{2}^{+}}\left(\eta_{2}^{(\alpha)}\left(\xi, x_{2}\right)\right)^{2} h_{1} h_{2}\right)^{1 / 2}+\left(\sum_{\omega^{(\alpha)}}\left(\eta_{3}^{(\alpha)}(x)\right)^{2} h_{1} h_{2}\right)^{1 / 2} \\
& \left.\left.+\left(\sum_{\omega_{2}}\left(\eta_{3}^{(\alpha)}\left(\xi, x_{2}\right)\right)^{2} h_{1} h_{2}\right)^{1 / 2}\right]+\left(\sum_{\omega_{2}}\left(\eta_{4}\left(x_{2}\right)\right)^{2} h_{1} h_{2}\right)^{1 / 2}\right\} .
\end{aligned}
$$


To estimate the left hand side of the latter inequality in terms of parameter $h$ and to obtain an estimate for the convergence rate of approximations w.r.t. state, it is sufficient to estimate quantities (17):

$$
\begin{array}{ll}
\sum_{\omega_{1}^{(\alpha)+}} \sum_{\omega_{2}}\left(\eta_{1}^{(\alpha)}(x)\right)^{2} h_{1} h_{2} \leqslant M^{2}\left\|k_{1}^{(\alpha)}\right\|_{L_{\infty}\left(\Omega_{\alpha}\right)}^{2}|h|^{2}\left\|u_{\alpha}\right\|_{W_{2}^{2}\left(\Omega_{\alpha}\right)}^{2}, & \alpha=1,2 ; \\
\sum_{\omega_{1}^{(\alpha)}} \sum_{\omega_{2}^{+}}\left(\eta_{2}^{(\alpha)}(x)\right)^{2} h_{1} h_{2} \leqslant M^{2}\left\|k_{2}^{(\alpha)}\right\|_{L_{\infty}\left(\Omega_{\alpha}\right)}^{2}|h|^{2}\left\|u_{\alpha}\right\|_{W_{2}^{2}\left(\Omega_{\alpha}\right)}^{2}, & \alpha=1,2 ; \\
\sum_{\omega_{2}^{+}}\left(\eta_{2}^{(\alpha)}\left(\xi, x_{2}\right)\right)^{2} h_{1} h_{2} \leqslant M^{2}\left\|k_{2}^{(\alpha)}\right\|_{L_{\infty}\left(\Omega_{\alpha}\right)}^{2}|h|^{2}\left\|u_{\alpha}\right\|_{W_{2}^{2}\left(\Omega_{\alpha}\right)}^{2}, & \alpha=1,2 ; \\
\sum_{\omega^{(\alpha)}}\left(\eta_{3}^{(\alpha)}(x)\right)^{2} h_{1} h_{2} \leqslant M^{2} L^{2}\left\|d_{\alpha}\right\|_{L_{\infty}\left(\Omega_{\alpha}\right)}^{2}|h|^{2}\left\|u_{\alpha}\right\|_{W_{2}^{2}\left(\Omega_{\alpha}\right)}^{2}, & \alpha=1,2 ; \\
\sum_{\omega_{2}}\left(\eta_{3}^{(\alpha)}\left(\xi, x_{2}\right)\right)^{2} h_{1} h_{2} \leqslant M^{2} L^{2}\left\|d_{\alpha}\right\|_{L_{\infty}\left(\Omega_{\alpha}\right)}^{2}|h|^{2}\left\|u_{\alpha}\right\|_{W_{2}^{2}\left(\Omega_{\alpha}\right)}^{2}, & \alpha=1,2 ; \\
\sum_{\omega_{2}} \eta_{4}^{2}\left(x_{2}\right) h_{2} \leqslant M^{2}\left[h_{2}^{2}\|\theta\|_{L_{\infty}\left(0, l_{2}\right)}^{2}+\left\|S^{x_{2}} \theta-\Phi_{h}\right\|_{L_{\infty}\left(\omega_{2}\right)}^{2}\right] \sum_{k=1}^{2}\left\|u_{k}\right\|_{W_{2}^{2}\left(\Omega_{\alpha}\right)}^{2} &
\end{array}
$$

They are based on some cumbersome calculations and this is why we restrict ourselves by proving, say, the first of the estimates as $\alpha=1$. It follows from the chain of inequalities

$$
\begin{aligned}
\left|\eta_{1}^{(1)}(x)\right|= & \frac{1}{h_{1} h_{2}} \int_{x_{1}-h_{1}}^{x_{1}} \int_{e_{2}\left(x_{2}\right)} k_{1}^{(1)}\left(x_{1}-0.5 h_{1}, r_{2}\right)\left[\int_{x_{1}-0.5 h_{1}}^{r_{1}} \frac{\partial^{2} u_{1}\left(m, r_{2}\right)}{\partial m^{2}} d m\right. \\
& \left.-\int_{x_{2}}^{r_{2}} \frac{\partial^{2} u_{1}\left(r_{1}, s\right)}{\partial r_{1} \partial s} d s\right] d r_{1} d r_{2} \mid \leqslant\left(h_{1} h_{2}\right)^{-1 / 2}\left\|k_{1}^{(1)}\right\|_{L_{\infty}\left(\Omega_{1}\right)}\left[h _ { 1 } \left(\int_{x_{1}-h_{1}}^{x_{1}}\right.\right. \\
& \left.\left.\int_{e_{2}\left(x_{2}\right)}\left|\frac{\partial^{2} u_{1}\left(m, r_{2}\right)}{\partial m^{2}}\right|^{2} d m d r_{2}\right)^{1 / 2}+h_{2}\left(\int_{x_{1}-h_{1}}^{x_{1}} \int_{e_{2}\left(x_{2}\right)}\left|\frac{\partial^{2} u_{1}\left(r_{1}, s\right)}{\partial r_{1} \partial s}\right|^{2} d r_{1} d s\right)^{1 / 2}\right] \\
\leqslant & 2^{1 / 2}\left\|k_{1}^{(1)}\right\|_{L_{\infty}\left(\Omega_{1}\right)}|h|\left(h_{1} h_{2}\right)^{-1 / 2}\left\|u_{1}\right\|_{W_{2}^{2}\left(\left(x_{1}-h_{1}, x_{2}\right) \times e_{2}\left(x_{2}\right)\right)}, \quad x \in \omega_{1}^{(1)+} \times \omega_{2}
\end{aligned}
$$

which can be easily proved. The proof is complete.

Remark 2. On the basis of accuracy estimates for approximations w.r.t. state established in the present work, in future we shall study convergence of approximations w.r.t. functional, control, approximations regularizations.

\section{BIBLIOGRAPHY}

1. F.P. Vasil'ev. Optimization methods. Factorial Press, Moscow (2002). (in Russian).

2. A.Z. Ishmukhametov. Issues of stability and approximations of optimal control problems. Comp. Center RAS, Moscow (1999). (in Russian).

3. A.Z. Ishmukhametov. Issues of stability and approximations of optimal control problems with distributed parameters. Comp. Center RAS, Moscow (2001). (in Russian).

4. M.M. Potapov. Approximation of extremum problems in mathematical physics (hyperbolic equations), Moscow State Univ. Press, Moscow (1985). (in Russian).

5. F.V. Lubyshev. Difference approximations of optimal control problems by systems described by partial differential equations. Bashkir State Univ. Press, Ufa (1999). (in Russian). 
6. A.A. Samarskii, V.B. Andreev. Difference methods for elliptic equations. Nauka, Moscow (1976). (in Russian).

7. A.A. Samarskii. Theory of difference schemes. Nauka, Moscow (1989). (in Russian).

8. V.A. Tsurko. The accuracy of finite-difference schemes for parabolic equations with a discontinuous solution // Diff. Uravn. 36:7, 986-992 (2000). [Diff. Equats. 36:7, 1094-1101 (2000).]

9. V.A. Tsurko. Finite-difference methods for convection-diffusion problems with discontinuous coefficients and solutions // Diff. Uravn. 41:2, 274-280 (2005). [Diff. Equats. 41:2, 290-296 (2005).]

10. F.V. Lubyshev. Approximation and regularization of optimal control problems for parabolic equations with controls in coefficients // Dokl. RAN. 349:5, 598-602 (1996). [Dokl. Math. 54:1, 589-593 (1996).]

11. F.V. Lubyshev, M.É. Fairuzov. Approximation and regularization of optimal control problems for quasilinear elliptic equations // Zhurn. Vych. Matem. Matem. Fiz. 41:8, 1148-1164 (2001). [Comput. Math. Math. Phys. 41:8, 1092-1107 (2001).]

12. F.V. Lubyshev, A.R. Manapova. On some optimal control problems and their finite difference approximations and regularization for quasilinear elliptic equations with controls in the coefficients // Zhurn. Vych. Matem. Matem. Fiz. 47:3, 376-396 (2007). [Comput. Math. Math. Phys. 47:3, 361-380 (2007).]

13. F.V. Lubyshev, A.R. Manapova. Difference approximations of optimization problems for semilinear elliptic equations in a convex domain with controls in the coefficients multiplying the highest derivatives // Zhurn. Vych. Matem. Matem. Fiz. 53:1, 20-46 (2013). [Comput. Math. Math. Phys. 53:1, 8-33 (2013)]

14. E.M. Kartashov. Analytic methods in heat theory for solid bodies. Vysshays shkola, Moscow (1985). (in Russian).

15. S.L. Sobolev. Some applications of functional analysis in mathematical physics. Siberian Branch of AS USSR, Novosibirsk (1962). (in Russian).

16. O.A. Ladyzhenskaya. The boundary value problems of mathematical physics. Nauka, Moscow (1973). [Applied Mathematical Sciences, V. 49. Springer, NY (1985).]

17. H. Gaewski, K. Gröger, K. Zacharius. Nichtlineare operatorgleichungen und operatordifferentialgleichungen. Akademie-Verlag, Berlin (1974).

18. S. Fučik, A. Kufner. Nonlinear differential equations. Studies in Applied Mechanics, V. 2. Elsevier Scientific Publ. Co., Amsterdam (1980).

19. D. Gilbarg, N.S. Trudinger. Elliptic partial differential equations of second order. Springer, Berlin (1983).

20. K. Rektorys. Variational methods in mathematics, science and engineering. D. Reidel Publishing Company, Dordrecht, (1980).

21. F.E. Browder. Materials to joint soviet-american simposium on partial differential equations. Novosibirsk (1963). (in Russian).

22. N.T. Drenska. Accuracy of numerical algorithms for the one-dimensional problem of cooling of metals in molds // Vestnik Mosk. Univ. Ser. 15. Vych. Matem. Kibern. 4, 15-21 (1981). [Mosc. Univ. Comput. Math. Cybern. 4, 17-24 (1981).]

Aygul' Rashitovna Manapova,

Bashkir State University,

Z. Validi str., 32,

450074, Ufa, Russia

E-mail: aygulrm@mail.ru

Fedor Vladimirovich Lubyshev,

Bashkir State University,

Z. Validi str., 32,

450074, Ufa, Russia

E-mail: aygulrm@mail.ru 